\title{
Development and In Vitro Characterization of a Gemcitabine-loaded MUC4-targeted Immunoliposome Against Pancreatic Ductal Adenocarcinoma
}

\author{
CARLOS UREY, KATARZYMA SAID HILMERSSON, BODIL ANDERSSON, \\ DANIEL ANSARI and ROLAND ANDERSSON
}

Department of Surgery, Clinical Sciences Lund, Skane University Hospital, Lund University, Lund, Sweden

\begin{abstract}
Background/Aim: Pancreatic Ductal adenocarcinoma (PDAC) is a devastating disease. Gemcitabine is the standard chemotherapeutic agent against PDAC but has only limited effectiveness. The aim of the study was to develop and study the targeting affinity and in vitro antiproliferative effect of a MUC4-targeted gemcitabineloaded immuno-liposome for treatment of PDAC. Materials and Methods: Gemcitabine-loaded immunoliposomes were developed by grafting anti-MUC4 antibodies to the liposomal surface. Targeting affinity was compared in vitro between immunoliposomes and non-targeted liposomes and anti-proliferative effect was compared in vitro between free drug, non-targeted liposomal gemcitabine and MUC4targeted immunoliposomal gemcitabine on a MUC4-positive pancreatic cancer cell line, Capan-1. Results: Development of a MUC4-targeted immunoliposome was confirmed and characterized by immunoblots and size characterization. The MUC4-targeted immunoliposome showed a significantly higher targeting affinity compared to the non-targeted liposomes and also showed an improved antiproliferative effect compared to free and non-targeted liposomal drug. Conclusion: Successful development and characterization of a MUC4-targeted immunoliposome shows promising results for a targeted treatment and improved retention of gemcitabine for treatment of PDAC.
\end{abstract}

This article is freely accessible online.

Correspondence to: Roland Andersson, Professor of Surgery, Department of Surgery, Clinical Sciences Lund, Skane University Hospital, SE-22185 Lund, Sweden. Tel: +4646172359 e-mail: roland.andersson@med.lu.se

Key Words: Immunoliposomes, pancreatic ductal adenocarcinoma, MUC4, Gemcitabine, Capan-1, drug delivery.
Pancreatic ductal adenocarcinoma (PDAC) is an aggressive and devastating disease. In the US and the European countries, PDAC is the fourth leading cause of cancer-related death $(1,2)$. Incidence and mortality rates follow closely $(3$, 4) and even though the relative 5-year survival rate has improved slightly over the last years, it is still as low as 7\% (2). Surgically removing the primary tumor, i.e. resection, is the only potential cure for PDAC. However, at the time of diagnosis, only around $20 \%$ of patients are eligible for surgical resection (5), and even so, most patients will relapse within five years (6). For patients with non-resectable tumors, the main treatment option is chemotherapy which mainly consists of a gemcitabine-based regimen, either alone or in combination with other drugs (7).

Mucins belong to a large family of high molecular weight glycoproteins characterized by long tandem repeat regions rich in serine, proline and threonine (8). Mucins are expressed by various epithelial cells (9) while the human mucin family consists of 21 members, MUC1-MUC21. Based on their functions, mucins are further divided into two different subclasses, as either secreted or membrane-bound mucins (10). Secreted mucins can be divided into gelforming (MUC2, MUC5AC, MUC5B, MUC6) and non-gelforming (MUC7) (9). The secreted mucins form a physical barrier of mucous gel for protection of epithelial cells in the respiratory and GI tracts. Membrane-bound mucins (MUC1, MUC3A, MUC3B, MUC4, MUC12, MUC13, MUC15, MUC16 and MUC17) are composed of a cytoplasmic tail and a transmembrane region and they contribute to the protective mucous gel through their single extracellular domain of O-glycosylated tandem repeats $(8,10)$.

In contrast to a healthy pancreas, which has weak or no mucin expression, pancreatic cancer is characterized by an aberrant expression of multiple mucins. During progression from normal pancreas to a malignant state, expression and glycosylation of both secreted and membrane-bound mucins are altered (10). Furthermore, altered expression of mucins is 
reported to facilitate tumor growth, proliferation, invasion, differentiation and immune recognition $(8,10,11)$. MUC4 is de novo expressed in pancreatic tumors as well in early pancreatic lesions (such as pancreatic intraepithelial neoplasia and intraductal papillary mucinous neoplasm) with its expression gradually increasing with disease progression and subsequent metastasis $(10,12)$. Furthermore, expression of mucins, and MUC4 in particular, has been related to resistance against Gemcitabine and other nucleoside analogues in pancreatic cancer cells (13-17). In a clinical setting, we were able to show that low expression of MUC4, compared to high MUC4 expression, confers a survival benefit for patients with resectable pancreatic tumors receiving adjuvant gemcitabine (18), further strengthening the role of MUC4 as a potential predictor of treatment response and a predictive biomarker.

Gemcitabine (2',2'-difluorodeoxycytidine, dFdC), a nucleoside analogue, is today the standard chemotherapy for PDAC in both adjuvant and palliative therapy. Inside the cell, Gemcitabine undergoes a series of phosphorylation steps before incorporating itself into DNA during replication, disrupting the replication process and ultimately inducing cellular apoptosis; a mechanism also known as "masked-chain termination" (19). Gemcitabine, despite being the standard treatment, is not devoid of limitations. Despite the inherent and acquired resistance mechanisms of pancreatic tumor cells towards gemcitabine, it has a very short half-life of around 15 min $(20,21)$, induces systemic toxicity (22), and undergoes a rapid conversion into its inactive metabolite by cytidine deaminase in the blood stream (23). Another major limitation of gemcitabine is its need for active cellular uptake by the hENT1 receptors on the surface of pancreatic cancer cells. Studies have shown that cells with low hENT1 expression are resistant towards gemcitabine $(24,25)$, and even more concerning, only one third of all PDAC patients overexpress hENT1, further limiting the use of gemcitabine (25-27).

During the last decade, many attempts have been made in order to improve gemcitabine's pharmacological profile. Some attempts have revolved around the development of a gemcitabine prodrug in order to improve its cellular uptake, whereas many more have focused on the delivery of gemcitabine through nanoparticle drug delivery systems, such as polymeric nanoparticles, micelles, and in particular liposomes (20). Liposomes are non-toxic, nanosized, artificial vesicles composed of a phospholipid bilayer with an aqueous interior. Liposomes are particularly popular as drug delivery systems due to their ability of entrapping hydrophilic, hydrophobic and amphiphilic drugs in their aqueous interior or lipid bilayer and thereby improving the drugs' pharmacokinetics and biodistribution profiles. This has been the case for FDA-approved drugs like liposomal doxorubicin (28), cisplatin (29) and irinotecan (30). Furthermore, in vitro studies have shown that liposomes are predominantly taken up by cells via endocytosis or fusion $(31,32)$, which, in the case of drugs like Gemcitabine, would help to increase their efficacy.

Liposomes with diameters of 70-200 $\mathrm{nm}$ have the advantage of passively accumulating in tumors due to the Enhanced Permeability and Retention (EPR) effect $(33,34)$. The EPR effect arises from leaky vasculature nearby tumor areas, allowing for the accumulation of particles below a certain diameter in tumor tissue. By coupling a targeting ligand to the liposomal surface an active targeting may be achieved. After extravasation into the tumor interstitial space, liposome retention and cellular internalization can be enhanced through the interaction between the targeting ligand and its target (35). A number of targeting ligands have been used in liposomes (36) with antibodies coupled to liposomes, i.e. immunoliposomes, being among the most common.

In this study, we developed and characterized an anti-MUC4 immunoliposome loaded with gemcitabine (iGemLip). We evaluated and compared the immunoliposome antiproliferative effect to that of the free drug and non-targeted liposomal Gemcitabine (GemLip), as well as the immunoliposome targeting ability compared to that of non-targeted liposomes in a MUC4-positive pancreatic cancer cell line.

\section{Materials and Methods}

Materials. Dipalmitoylphosphatidylcholine (DPPC), cholesterol (Chol), 1,2-distearoyl-sn-glycero-3-phosphoethanolamine-N-[metho$\mathrm{xy}$ (polyethylene glycol)-2000] (DSPE-mPEG ${ }_{2000}$ ) and 1,2-distearoyl-sn-glycero-3-phosphoethanolamine- $\mathrm{N}$-[maleimide(polyethylene glycol)-2000] (DSPE-PEG 2000 -Mal) were purchased from Avanti Polar Lipids (Alabaster, AL, USA). Texas Red-DHPE (Texas Red ${ }^{\circledR}$ 1,2-dihexadecanoyl-sn-glycero-3-phosphoethanolamine, triethylammonium salt) from Thermo Fisher, (Waltham, MA, USA) and HEPES, Gemcitabine $\mathrm{HCl}$ and Traut's reagent from Sigma Aldrich Co. (St. Louis, MO, USA).

Preparation of liposomes. Liposomes of DPPC:Chol:DSPE$\mathrm{mPEG}_{2000}$ or DPPC:Chol:DSPE-PEG 2000 -Mal at (80:20:5) molar ratios were prepared by the thin lipid hydration technique. When necessary, $0.1 \mathrm{~mol} \%$ of Texas Red- DHPE was also added to the lipid mixture. Briefly, phospholipids were dissolved in a round bottom flask using a $(3: 1 \mathrm{v} / \mathrm{v})$ mixture of chloroform:methanol. Organic solvents were removed, and a lipid film was formed under reduced pressure at $60^{\circ} \mathrm{C}$ using a Büchi Rotavapor for $1 \mathrm{~h}$. In order to completely remove traces of organic solvent, lipid films were further dried overnight in a Labconco Freezone Plus 6 lyophilizer (Labconco Corporation, Kansas City, MO, USA). Lipid films were hydrated with either Hepes Buffered Saline (HBS, pH 7.4) or a Gemcitabine solution under rotation at $60^{\circ} \mathrm{C}$ for $1 \mathrm{~h}$ and further vortexed until all the lipid film was dissolved. The suspension of vesicles was serially extruded 11 times through a $200 \mathrm{~nm}$ and $100 \mathrm{~nm}$ stacked pair of polycarbonate filters, each, to obtain a suspension of small liposomal vesicles. Non-encapsulated Gemcitabine was removed from solution by gel chromatography using a Sephadex G25 column (GE Healthcare Life Sciences AB, Uppsala, Sweden), and columns were equilibrated with HBS. 
Immunoliposome preparation. For the preparation of iGemLip, IgG antibody was covalently linked to liposomes via a thioether linkage between thiol groups of the antibody and the terminal Maleimidegroup on DSPE-PEG 2000 -Mal on the surface of the liposomes. Coupling was performed after the separation of non-encapsulated drug. Prior to liposome coupling, anti-MUC4 antibody (MABT395, clone 8G7, Merck Milipore, Billerica, MA, USA) was thiolated by incubating with Traut's reagent at a 1:10 molar ratio IgG:Traut's reagent for $1 \mathrm{~h}$ at RT. Then, non-reacted Traut's reagent was removed from antibody by gel chromatography using a PD Spintrap G-25 (GE Healthcare, Uppsala, Sweden) column according to the manufacturer's instructions. After antibody thiolation, antibodies were added to Gemcitabine liposomes at a 1:1,000 molar ratio of IgG:lipids in liposomes solution for $1 \mathrm{~h}$ at $60^{\circ} \mathrm{C}$. After incubation for $1 \mathrm{~h}$, cystein was added to a $1 \mathrm{mM}$ final concentration to quench unreacted maleimide groups. Finally, unreacted cysteine and unbound antibodies were removed from immunoliposomes by 30 min centrifugation at $4,000 \times g$ using a $300 \mathrm{kDa}$ MWCO Vivaspin2 centrifugal concentrator (Vivaproducts Inc., Littleton, MA, USA). After centrifugation and removal of unbound cysteine and antibodies, concentrated liposomal samples were diluted back to their original concentration $(1 \times)$.

Characterization of liposomes. For encapsulation efficiency, $10 \mu \mathrm{l}$ aliquots of liposomal gemcitabine, prior to and after removal of non-encapsulated gemcitabine, were diluted $100 \times$ in $\mathrm{MeOH}$. Gemcitabine concentration was measured with spectrophotometry at $269 \mathrm{~nm}$ absorption wavelength with a Multiskan Go plate reader using the SkanIt Software 3.2 (Thermo Fisher Scientific, Waltham, MA, USA). To calculate encapsulation efficiency (EE) and drug loading (DL), the following formula was used:

$$
E E(\%)=\frac{A_{1}}{A_{0}} \times 100 \quad(1), \quad D L(\%)=\frac{W_{D}}{W_{L}} \times 100
$$

For EE, $\mathrm{A}_{0}$ is the light absorbance for the total amount of drug added and $\mathrm{A} 1$ is the absorbance of drug remaining after removal of non-encapsulated drug. For DL, $\mathrm{W}_{\mathrm{D}}$ is the weight of the loaded drug and $\mathrm{WL}$ is the weight of the liposomes in moles.

Mean sizes of liposomes were determined by dynamic light scattering using a Zetasizer Nano SZ (Malvern Instruments, Spring Lane South, Worcestershire, UK) photo-correlation spectroscopy machine. Samples were diluted $(1: 100 \mathrm{v}: \mathrm{v})$ in a solution of liposomal sample: $\mathrm{dH} 2 \mathrm{O}$. Samples were measured 3 times each and dilutions were prepared in quartz cuvettes immediately before measurement. Mean sizes are presented as Z-averages from cumulant fit analyses of liposome samples. Material refractive index was set to 1.50 , medium refractive index was set to 1.33 and viscosity set at $0.887 \mathrm{cP}$ at $25^{\circ} \mathrm{C}$.

Antibody conjugation to liposomes was controlled using electrophoresis and Western Blot. Briefly, samples were loaded and electrophoresed onto a Mini-Protean TGX stain-free gel, (Bio-Rad Laboratories AB, Solna, Sweden) under non-reducing conditions using a Native Sample buffer (Bio-Rad Laboratories AB, Solna, Sweden) and a Tris/Glycine buffer for native gels (25 mM Tris, 192 $\mathrm{mM}$ Glycine, $\mathrm{pH}$ 8.3). After electrophoresis, samples were blotted/transferred on a Trans-Blot Turbo Transfer System (Bio-Rad Laboratories AB, Solna, Sweden) followed by blocking in 5\% (w/v) milk in Tris-Buffered Saline Tween-20 for $1 \mathrm{~h}$ in RT. The membranes were then washed and incubated for $1 \mathrm{~h}$ at $4^{\circ} \mathrm{C}$ with secondary antibody (anti-mouse IgG HRP-linked Ab; A4416, Sigma Aldrich, St. Louis, MO, USA). Chemiluminescence detection was performed using SuperSignal West FEMTO substrate (Pierce Thermo Scientific, Waltham, MA, USA) and immunoblots were visualized by LI-COR Odyssey Fc Imager (LI-COR Biosciences, Lincoln, NE, USA) and Image Studio (v.3.1.4; LI-COR Sciences, Lincoln, NE, USA).

Cell culture. The human pancreatic carcinoma cell line Capan-1 was provided by Professor Surinder Batra (University of Nebraska Medical Center, Omaha, Nebraska, USA). Capan-1 cells were maintained in Iscove's modified Dulbecco's medium (Gibco, Life Technologies, Grand Island, NY, USA) supplemented with $20 \%$ fetal bovine serum (FBS; Gibco, Life Technologies, Grand Island, NY, USA). Cell lines were also supplemented with antibiotics (100 U/ml penicillin and $100 \mu \mathrm{g} / \mathrm{ml}$ streptomycin) (Gibco, Life Technologies, Grand Island, NY, USA). Cells were grown in T-75 culturing flasks (Sarstedt, Nümbecht, Germany) and kept in a humidified $5 \% \mathrm{CO} 2$ atmosphere at $37^{\circ} \mathrm{C}$. Culturing cell media was changed twice a week and cells were passaged once a week before reaching confluence. Before experiments, cells were washed with Dulbecco's phosphate-buffered saline without $\mathrm{Ca}^{+}$or $\mathrm{Mg}^{2+}(\mathrm{PBS}$; Gibco, Life Technologies, Grand Island, NY, USA)). Capan-1 cells were detached using TrypLE Select (Thermo Fisher Scientific, Waltham, MA, USA) for 10 min before being harvested and pelleted at 1,200 rpm for $4 \mathrm{~min}$. After dissociation of cell pellet by gentle pipetting, cell concentration and viability were determined using $0.06 \%$ trypan blue (Thermo Fisher Scientific, Waltham, MA, USA).

Cellular uptake and binding assay of liposomes. For the liposomal binding assay, Capan- 1 cells were seeded $\left(5 \times 10^{3}\right.$ cells/well) in 96well plates for fluorescent reading (Vision plate, 4titude, Surrey, UK) in standard culturing medium for $24 \mathrm{~h}$ at $37^{\circ} \mathrm{C}$ to allow cell adhesion. After the initial $24 \mathrm{~h}$, cell medium was replaced with fresh medium containing fluorescently labeled $(0.1 \mathrm{~mol} \%$ Texas RedDHPE) GemLip or iGemLip at different concentrations. After $1 \mathrm{~h}$ or 4h, cellular medium containing fluorescently labeled liposomes was removed, cells were washed twice with PBS and fresh medium was added. Fluorescence from cells was measured with a 496/519 excitation/emission filter using a PheraStar FS plate reader with PHERAstar Mars version 2.10 R3 software (BMG, Offenburg, Germany). For each plate, cells which were incubated only with fresh medium were used as blanks. For each liposomal concentration, $\mathrm{n}=10$.

Furthermore, to study cellular uptake, Capan-1 cells were seeded $\left(20 \times 10^{3}\right.$ cells/chamber $)$ in 8 -chamber slides in standard culturing medium for $24 \mathrm{~h}$ at $37^{\circ} \mathrm{C}$ to allow cell adhesion. After the first 24 $\mathrm{h}$, cellular medium was replaced with fresh cellular medium containing fluorescently labeled liposomes at different concentrations. On each chamber slide, two slides were used as blanks that were only treated with fresh medium and another two slides were treated with non-fluorescent liposomes. Cells were then incubated with fluorescently labeled liposomes for $1 \mathrm{~h}$ and $4 \mathrm{~h}$ at $37^{\circ} \mathrm{C}$, then cells were washed three times with ice-cold PBS before being fixated with $2 \%$ paraformaldehyde in PBS for $20 \mathrm{~min}$. After being washed three times with ice-cold PBS, cell nuclei were stained with DAPI (Life Technologies, Thermo Fisher Scientific, Waltham, MA, USA) for $10 \mathrm{~min}$. Fluorescence images were analyzed using a Nikon Eclipse 80i microscope with a Nikon DSQi1 camera and NIS-Elements software. 
Cell viability assay. To assess cell viability, the WST-1 (Roche Life Science, Indianapolis, Indiana, USA) cell proliferation assay was carried out.WST-1 is based on the reduction of a tetrazolium salt to a soluble derivative. For the experiments, Capan- 1 cells were seeded $\left(5 \times 10^{3}\right.$ cells/well) in 96 -well plates in standard culturing medium for $24 \mathrm{~h}$ at $37^{\circ} \mathrm{C}$ to allow cell adhesion. After $24 \mathrm{~h}$, the cell medium was replaced with fresh medium containing either Gemcitabine, GemLip or iGemLip at different concentrations. For each plate, untreated cells which only received fresh medium were used as controls. Following a $24 \mathrm{~h}$ incubation with treatments, cell medium was removed and cells were washed twice with PBS before adding fresh medium. Cells were further incubated for $24 \mathrm{~h}$ and $48 \mathrm{~h}$ before adding $10 \mu \mathrm{l}$ WST-solution to each well. After $4 \mathrm{~h}$, samples were measured on a Multiskan GO plate reader (test wavelength $450 \mathrm{~nm}$, reference wavelength 660 $\mathrm{nm}$ ) using the SkanIt Software 3.2 (both from Thermo Fisher Scientific, Waltham, MA, USA).

Statistical analysis. Proliferation and cell binding assay data are expressed as means \pm SD of six and 10 replicate wells per plate, respectively. Statistical analyses were performed by one-way ANOVA and Student $t$-test using GraphPad Prism software (GraphPad Software Inc., La Jolla, CA, USA). A $p$-value of $\leq 0.05$ was considered statistically significant.

\section{Results}

Liposome and immunoliposome preparation and characterization. Liposomes were produced by the thinlipid film hydration method and gemcitabine was encapsulated by a passive encapsulation method. Mean particle size of GemLip and iGemLip were $129 \mathrm{~nm}$ and 168 $\mathrm{nm}$, respectively. These values indicate that the grafting of antibodies to liposomes have an impact on size, increasing liposomal sizes by nearly $40 \mathrm{~nm}$. The mean gemcitabine encapsulation efficiencies (EE) for GemLip and iGemLip were $7.7 \pm 3.4$ and $5.6 \pm 0.19 \%(n=3)$, respectively. Mean drug loading (DL) for GemLip and iGemLip was 29.5 \pm 13.1 and $21.6 \pm 0.73 \%(n=3)$, respectively, showing no significant difference in either EE or DL between both liposomal preparations.

Immunoliposomes were prepared by the conventional antibody coupling method after thiolation of antibodies. Conjugation of the monoclonal antibody to liposomes was confirmed by western blot. An IgG antibody has a weight of $150 \mathrm{kDa}$. Upon covalent binding of the antibody to the distal end of the DSPE-PEG 2000 -Mal, the molecular weight of the antibody should increase due to the added weight of DSPE$\mathrm{PEG}_{2000}$-Mal. As shown in Figure 1, the band corresponding to the thiolated anti-MUC4 antibody is present at $150 \mathrm{kDa}$, whereas the band for the antibodies conjugated to iGemLip indicate a higher molecular weight indicating that the antiMUC4 antibody was efficiently coupled to the liposomes. As a control, GemLip were also assayed to rule out falsepositives. As can be seen in lane 4 of Figure 1, no signal from GemLip could be detected.

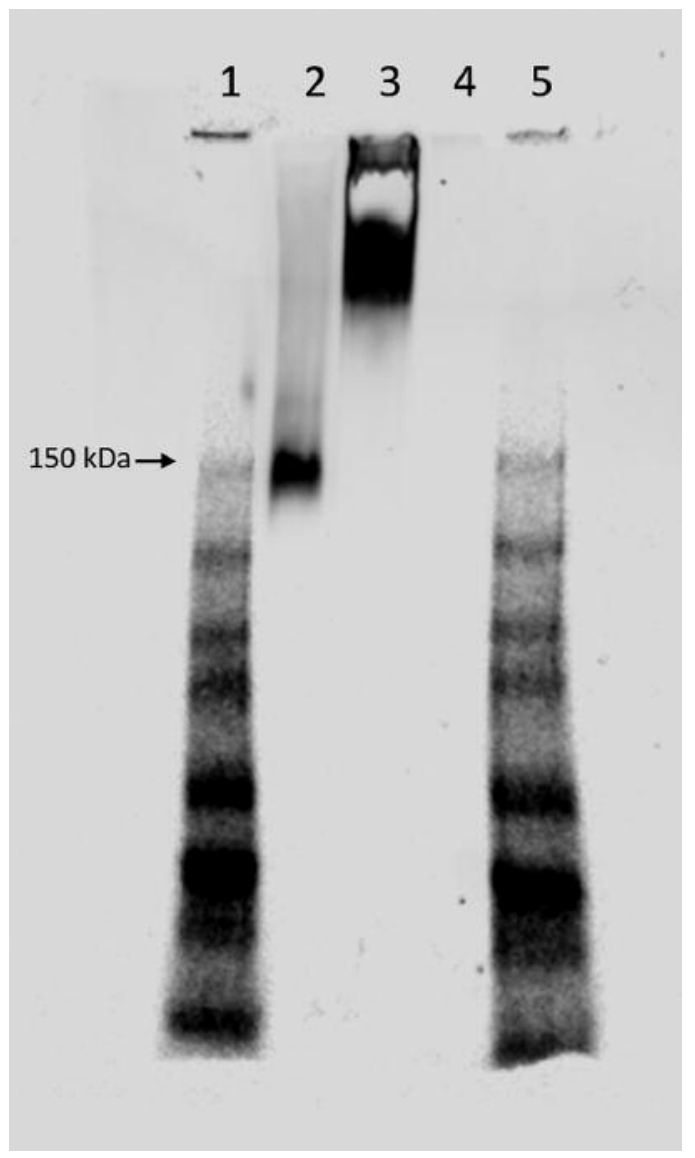

Figure 1. Confirmation of anti-MUC4 antibody conjugation to liposomes. Conjugation of antibodies was confirmed by SDS-Page. Lane 1 and 5: full range ladders, lane 2: thiolated anti-MUC4 $\operatorname{IgG}$, lane 3: Gemcitabine immunoliposomes after removal of free antibodies, and lane 4: Gemcitabine non-targeted liposomes.

Cellular uptake and binding assays of liposomes. Cellular binding and uptake of non-targeted liposomes and immunoliposomes was quantified by fluorescence reading and visualized by fluorescence microscopy.

Fluorescently labeled GemLip and iGemLip were incubated at different lipid concentrations with Capan-1 cells for $1 \mathrm{~h}$ and $4 \mathrm{~h}$ for fluorescence microscopy. As shown in Figure 2, fluorescence microscopy imaging shows that there is a clear increase of binding of fluorescently labeled iGemLip to Capan-1 cells compared to GemLip. This is true for the two lipid concentrations used for both incubation times. Cells were also incubated with regular, non-fluorescent, liposomes at the highest lipid concentration of $500 \mu \mathrm{M}$ lipid. No fluorescent signal could be detected for any of the two time points.

Following these observations, the binding affinity of immunoliposomes and non-targeted liposomes were tested. To this end, fluorescently labeled liposomes were incubated 
A

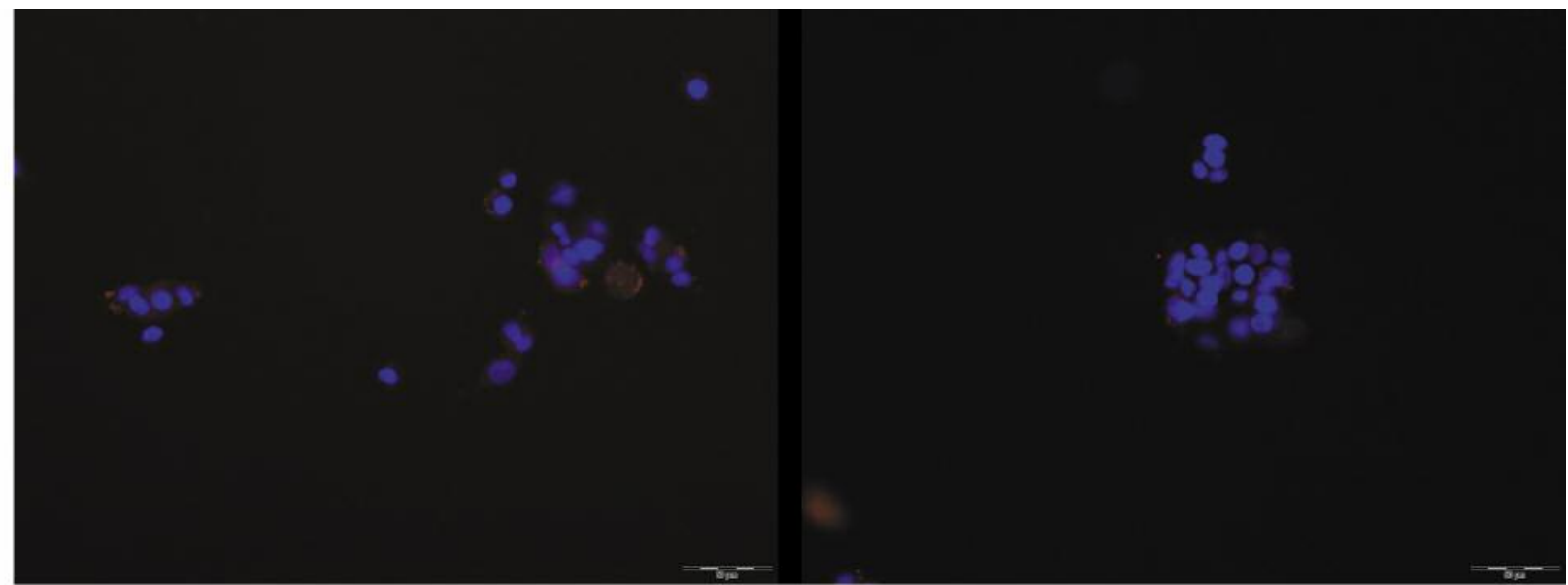

B

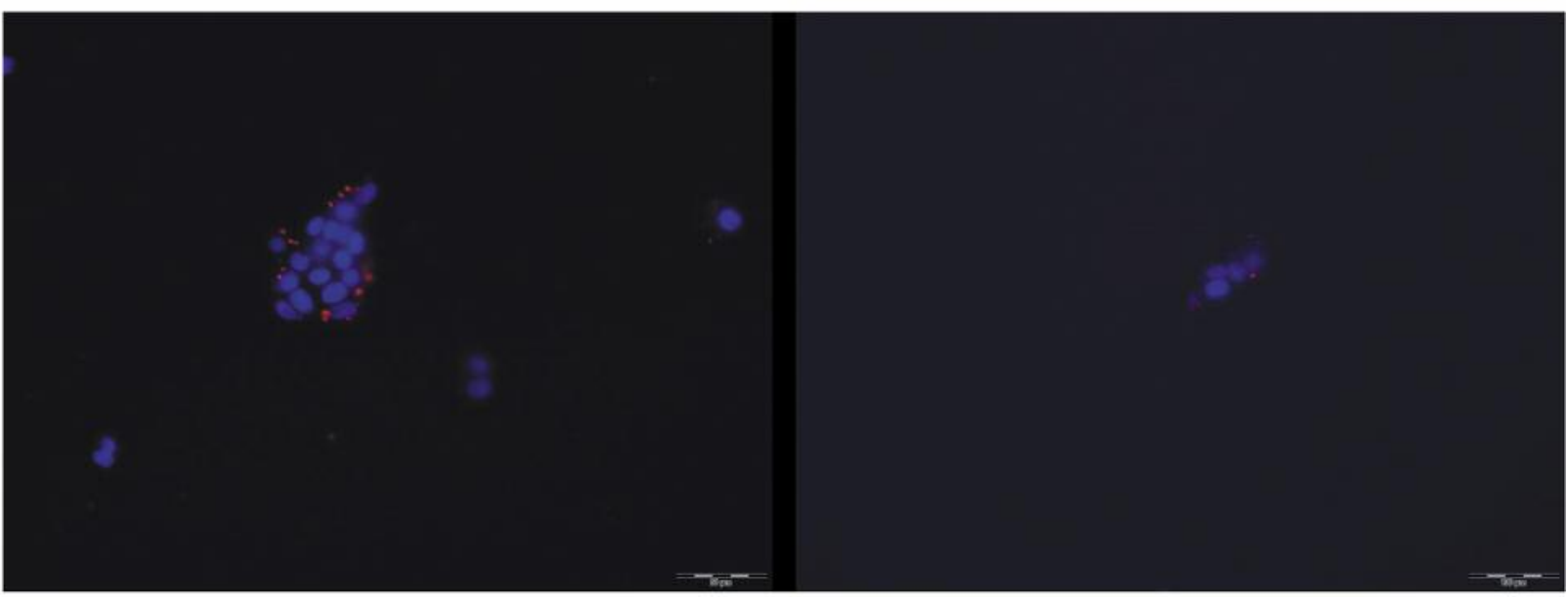

Figure 2. Increased cellular uptake of iGemLip to Capan-1 compared to GemLip. Fluorescence microscopy images from cell uptake study of fluorescently-labeled iGemLip (left panel) and GemLip (right panel) after (A) $1 \mathrm{~h}$ and (B) $4 \mathrm{~h}$ incubation with Capan-1 cells at $37^{\circ} \mathrm{C}$. The images depict cells treated with $500 \mu \mathrm{M}$ lipid concentration. Cell nuclei are colored blue and red depicts liposomes taken up or adsorbed to cells. iGemLip exhibits an increased cellular uptake compared to GemLip after both 1 h and $4 \mathrm{~h}$ incubation times.

with Capan-1 cells at three different lipid concentrations for $1 \mathrm{~h}$ and $4 \mathrm{~h}$. As the fluorescence reading from cells show (Figure 3), binding of both iGemLip and GemLip is dosedependent. Even so, liposome binding affinity is significantly higher for iGemLip compared to GemLip for all concentrations and both incubation times.

Cellular viability assay. Cell viability of Gemcitabine immunoliposomes was compared to that of liposomal gemcitabine and free gemcitabine on Capan-1 cells. For all treatments, cells were incubated with three different concentrations of Gemcitabine for $24 \mathrm{~h}$ before removing the treatment and incubating cells for further $24 \mathrm{~h}$ and 48 h (Figure 4). Data was normalized to untreated controls on each plate and cell viability is presented as "\% of Control". Surprisingly, a certain resistance to the different
Gemcitabine treatments was shown by the Capan-1 cell line as cell viability levels remained relatively constant within the range of $1-100 \mu \mathrm{M}$ gemcitabine. For the two lower concentrations, for both $24 \mathrm{~h}$ and $48 \mathrm{~h}$, no significant difference in cell viability is seen for the three different treatments. However, at the highest treatment concentration, after both $24 \mathrm{~h}$ and $48 \mathrm{~h}$, the anti-MUC4 immunoliposomes exhibited a significantly increased cytotoxic effect compared to both free drug and liposomal gemcitabine. Furthermore, cells were also treated with the highest lipid concentration of empty non-targeted liposomes and immunoliposomes for $24 \mathrm{~h}$ and $48 \mathrm{~h}$. Empty vehicles showed no significant impairment of cell viability meaning that the drug-free non-targeted liposomes and immunoliposomes are non-toxic to cells at the administered concentrations used in this study. 


\section{Discussion}

In this work, we set out to develop and characterize a gemcitabine-loaded MUC4-targeted immunoliposome to study if, and to what extent, MUC4 could be used as a target for improved delivery and retention of Gemcitabine in pancreatic cancer treatment.

Gemcitabine, which is the most commonly used anticancer drug for treatment of PDAC, does not infrequently fail to provide the desired therapeutic effect (20-25). Due to the many limitations and resistance factors of Gemcitabine, repeated attempts have been made in order to improve its therapeutic efficacy (20), also involving the use of liposomes. Liposomes have gained a widespread use during the last decades and a series of FDA-approved liposomal drugs serve as proof of liposomes being able to improve a drugs pharmacokinetic profile (28-30). Furthermore, by conjugating antibodies to the liposomal surface, i.e. immunoliposomes, an active targeting and improved retention of a drug is possible if a suitable target is chosen (37).

Mucins, that belong to a family of large O-glycosylated proteins, serve mainly as a protective barrier for epithelial cells, but also play an important role in communication between cells and the matrix. Mucins have, however, also been found to function as tumor cell modulators with MUC4 being one of these $(10,38)$. MUC4 is not expressed in the healthy pancreas, but is aberrantly expressed in pancreatic cancer. Expression of MUC4 is observed in early lesions and its expression is seen to increase with disease progression and subsequent metastasis (10-12).

For the purpose of creating a targeted treatment for improved delivery and retention of Gemcitabine, we chose to use immunoliposomes as our drug delivery system and MUC4 as our target of choice. First, we focused on the development of a stable Gemcitabine-loaded anti-MUC4 immunoliposome by use of the conventional antibody coupling method (39). Another method, post-insertion of antibodies coupled to micelles (39) was also tested, but as this method yielded a significant loss of encapsulated drug upon fusion of micelles, we decided to use the conventional method in this study. The coupling of the antibody was confirmed by Western Blot analysis where we could see that the band corresponding to the antibody was still present in the immunoliposomal sample after removal of non-conjugated antibodies. Also, the expected increase in molecular weight from binding to the PEG-chains was seen for the liposome-bound antibodies. Electrophoresis prior to WB was done under non-reducing conditions to prevent separation of antibodies into either two monovalent IgG's $(75 \mathrm{kDa})$ or two Fab fragments $(55 \mathrm{kDa})$. The nonreducing conditions also mean that antibodies from iGemLip were still conjugated to liposomes at the time of electrophoresis and hence, retaining the antibody in the gel and giving rise to the smearing seen on band 3 in Figure 3 .
Many drugs, like Doxorubicin (28), can actively be loaded into liposomes through an ammonium sulphate gradient. However, this approach is difficult to apply for gemcitabine due to its low pKa (3.6) and the fact that the majority of its molecules exist in a non-ionized state (40). Instead, a passive loading technique was employed from which we obtained loading with a high drug content, $\mathrm{DL}=21.6 \%$ for iGemLip. In order to achieve stable drug loading, as observed in other studies $(40,41)$, the intra-liposomal osmotic pressure (300 mOsm) was carefully matched to that of saline buffers on the exterior of the liposomes. By maintaining isotonicity after removal of free drug and buffer exchange to HBS, a burst release of Gemcitabine was prevented. Furthermore, we compared the size between GemLip and iGemLip to see if liposomal size was affected by antibody conjugation. Size was indeed affected and even though iGemLip was approx. $40 \mathrm{~nm}$ larger than GemLip with a diameter near $170 \mathrm{~nm}$, this should not affect pharmacokinetic properties as the size of iGemLip is still below the recommended $200 \mathrm{~nm}$ for in vivo use $(33,34)$.

After preparation and characterization of immunoliposomes, we proceeded to investigate the binding affinity of iGemLip compared to that of GemLip in the MUC4-positive pancreatic cancer cell line Capan-1 (42). For this purpose, we used two complementary techniques: fluorescence microscopy and fluorescence intensity readings. A Texas Red-fluorescent label was used to avoid false-positives from cellular autofluorescence. When using imaging with fluorescence microscopy, a clear cellular uptake was seen for both liposomal formulations, but it would appear that there was an increased binding to cells of iGemLip compared to GemLip. Further, these observations were confirmed by fluorescence readings of cellular fluorescence where we could see that, for all lipid concentrations at $1 \mathrm{~h}$ and $4 \mathrm{~h}$, binding of iGemLip to Capan-1 cells was significantly higher than binding of GemLip, confirming that we had an effective targeting of MUC4 from the iGemLip.

Finally, we investigated the antiproliferative effects of iGemLip and compared it to that of free Gemcitabine and non-targeted liposomal Gemcitabine, i.e. GemLip. In order to reflect a more realistic treatment effect, considering the short blood half-life of Gemcitabine, we decided to only treat cells for $24 \mathrm{~h}$ before removing treatment and further incubating cells for $24 \mathrm{~h}$ and $48 \mathrm{~h}$. For the two lowest concentrations used, the three treatments had a similar antiproliferative effect and it was only for the highest treatment concentration, for both $24 \mathrm{~h}$ and $48 \mathrm{~h}$, that iGemLip showed a small but significantly higher antiproliferative effect as compared to both the free drug and GemLip. Due to the drastically higher binding affinity of iGemLip to Capan-1 cells, this was a surprising result as we expected to see these results to be reflected to the cell viability studies. Even though iGemLip showed a clearly improved therapeutic efficacy compared to 


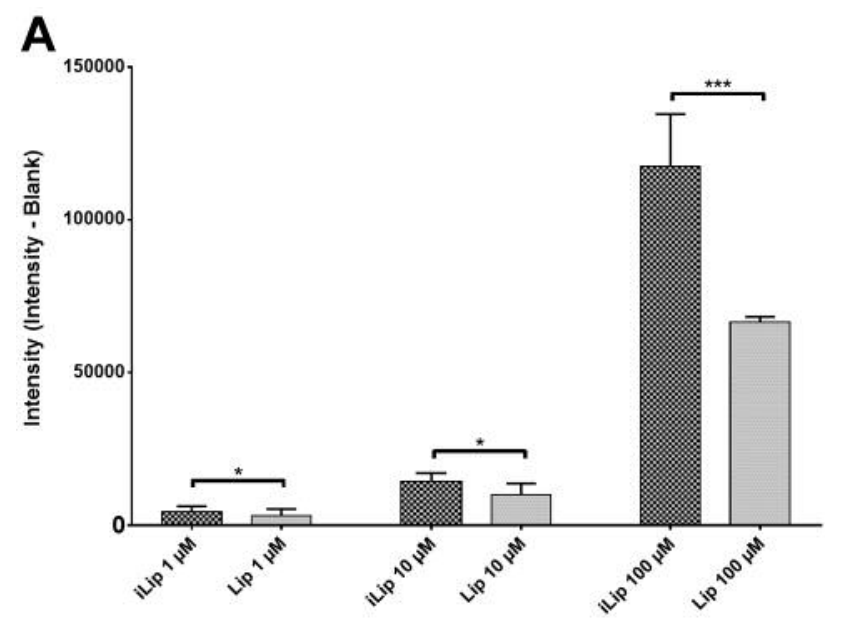

B

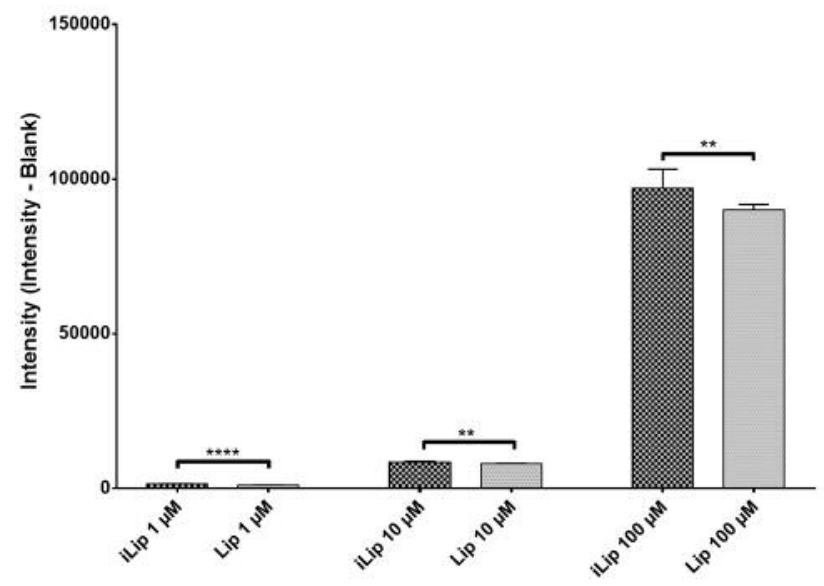

Figure 3. Increased binding affinity of iGemLip to Capan-1 cells than GemLip. Fluorescence intensity reading from cells from binding affinity of liposomes. Fluorescently-labeled iGemLip and GemLip were incubated with Capan-1 cells at $37^{\circ} \mathrm{C}$ for (A) $1 \mathrm{~h}$ and (B) $4 \mathrm{~h}$ at three different lipid concentrations. iGemLip exhibits a significantly increased binding affinity to Capan-1 cells compared to GemLip. Data are presented as mean $\pm S D . \quad(* p \leq 0.05, * * p \leq 0.01, * * * p \leq 0.001$ and $* * * * p \leq 0.0001)$.

the free drug, the improvement seen compared to GemLip was only marginal. There is the possibility that iGemLip has a higher adsorption to Capan-1cells, but that the fusion to cells or release and cellular uptake of drug remains similar for both iGemLip and GemLip. It has been proposed that the mucin mesh of cells acts as a barrier against nanoparticles in drug delivery; acting as a sieve, allowing the passage of small molecules, while delaying passage of larger particles. However, a recent study showed that with the use of liposomes one could overcome this barrier (43), and improve delivery of drug molecules to the target cells whereby the effect of the mucin mesh should not be an issue.
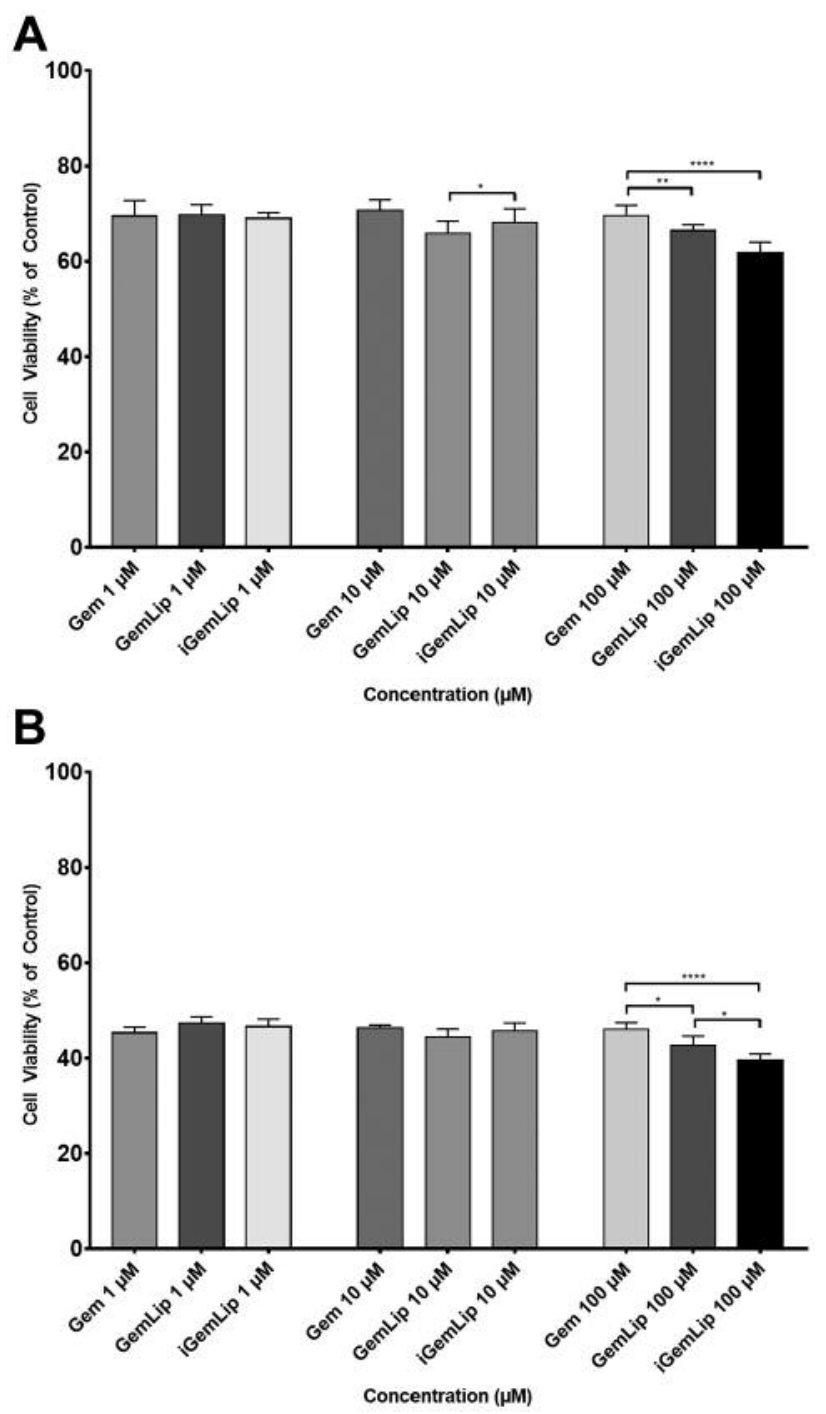

Figure 4. iGemLip has an increased antiproliferative effect on Capan-1 cells compared to free Gem and GemLip. Evaluating effect on cell viability of free Gemcitabine (Gem), GemLip and iGemLip in Capan-1 cells treated for (A) $24 \mathrm{~h}$ and (B) $48 \mathrm{~h}$ using a WST assay. iGemLip has a significantly higher antiproliferative effect on Capan-1 cells after both $24 \mathrm{~h}$ and $48 \mathrm{~h}$ compared to Gem and GemLip. The data are presented as mean $\pm S D .(* p \leq 0.05, * * p \leq 0.01, * * * p \leq 0.001$ and $* * * * p \leq 0.0001)$.

The targets for immunoliposomes should ideally be surface or membrane-bound antigens that have low or no expression in normal cells, but abundant expression in tumor cells. In this sense, MUC4 appears to be an ideal target due to its increased and retained expression throughout pancreatic cancer disease progression. Mucins have previously been used as targets in cancer treatments, mainly as vaccines or immunotherapy (44). However, the choice of 
MUC4 might be debatable as MUC4 expression has also been directly related to Gemcitabine resistance of pancreatic cancer cells, both in a preclinical and clinical setting (15-18). This could imply that a targeted gemcitabine-treatment might not be effective. On the other hand, the effective targeting of MUC4 opens up to other treatment possibilities, and especially point at a novel type of targeted treatment concept. The expression of MUC4 has been shown to promote proliferation, invasion and metastasis in pancreatic cancer cells (45). However, further in vitro studies showed that downregulation of MUC4 suppressed tumor cell growth and metastasis in pancreatic tumor cells (46), while other studies showed that MUC4 downregulation sensitized cells to gemcitabine treatment $(16,47)$. Combining these observations, this could be applied to a two-wave targeted treatment. First, MUC4-positive pancreatic cancer cells are targeted in an attempt to downregulate MUC4-expression, suppressing tumor cell growth and invasion while sensitizing pancreatic tumor cells to treatment, and then treating with Gemcitabine.

\section{Conclusion}

In conclusion, in the present study we successfully produced an immunoliposome targeted against MUC4 for improved delivery of gemcitabine to pancreatic cancer cells. The antiMUC4 immunoliposome, iGemLip, showed higher binding affinity to a MUC4-positive pancreatic cancer cell line, Capan-1, and also exhibited higher anti-proliferative effect compared to the free drug and non-targeted liposomal gemcitabine. This study shows promising results for a targeted delivery and improved retention of gemcitabine for treatment of pancreatic cancer. However, to fully elucidate the potential of this new targeted treatment, further studies are needed, in particular in an in vivo setting, before extending potential benefits of this type of treatment concept into use in the clinical setting.

\section{Aknowledgements}

The Authors would like to thank Dr. John Stegmayr at the Department of Laboratory Medicine, Lund University, Lund, Sweden for his technical assistance with fluorescence readings during cell-liposome binding assays.

\section{References}

1 Malvezzi M, Bertuccio P, Rosso T, Rota M, Levi F, La Vecchia $\mathrm{C}$ and Negri E: European cancer mortality predictions for the year 2015: does lung cancer have the highest death rate in EU women? Ann Oncol 26: 779-786, 2015.

2 Siegel RL, Miller KD and Jemal A: Cancer statistics, 2015. CA Cancer J Clin 65: 5-29, 2015.

3 Siegel R, Ma J, Zou Z and Jemal A: Cancer statistics, 2014. CA Cancer J Clin 64: 9-29, 2014.
4 Kamisawa T, Wood LD, Itoi T and Takaori K: Pancreatic cancer. Lancet 388: 73-85, 2016.

5 Malik NK, May KS, Chandrasekhar R, Wee W, Flaherty L, Iyer R, Gibbs J, Kuvshinoff B, Wilding G, Warren G and Yang GY: Treatment of locally advanced unresectable pancreatic cancer: a 10-year experience. J Gastrointest Oncol 3: 326-334, 2012.

6 Saif MW: Pancreatic neoplasm in 2011: an update. JOP 12: 316321, 2011.

7 Burris HA, 3rd, Moore MJ, Andersen J, Green MR, Rothenberg ML, Modiano MR, Cripps MC, Portenoy RK, Storniolo AM, Tarassoff P, Nelson R, Dorr FA, Stephens CD and Von Hoff DD: Improvements in survival and clinical benefit with gemcitabine as first-line therapy for patients with advanced pancreas cancer: a randomized trial. J Clin Oncol 15: 2403-2413, 1997.

8 Kufe DW: Mucins in cancer: function, prognosis and therapy. Nat Rev Cancer 9: 874-885, 2009.

9 Hollingsworth MA and Swanson BJ: Mucins in cancer: protection and control of the cell surface. Nat Rev Cancer 4: 45-60, 2004.

10 Kaur S, Kumar S, Momi N, Sasson AR and Batra SK: Mucins in pancreatic cancer and its microenvironment. Nat Rev Gastroenterol Hepatol 10: 607-620, 2013.

11 Terada T, Ohta T, Sasaki M, Nakanuma Y and Kim YS: Expression of MUC apomucins in normal pancreas and pancreatic tumours. J Pathol 180: 160-165, 1996.

12 Swartz MJ, Batra SK, Varshney GC, Hollingsworth MA, Yeo CJ, Cameron JL, Wilentz RE, Hruban RH and Argani P: MUC4 expression increases progressively in pancreatic intraepithelial neoplasia. Am J Clin Pathol 117: 791-796, 2002.

13 Bafna S, Kaur S, Momi N and Batra SK: Pancreatic cancer cells resistance to gemcitabine: the role of MUC4 mucin. Br J Cancer 101: 1155-1161, 2009.

14 Kalra AV and Campbell RB: Mucin overexpression limits the effectiveness of 5-FU by reducing intracellular drug uptake and antineoplastic drug effects in pancreatic tumours. Eur J Cancer 45: 164-173, 2009.

15 Skrypek N, Duchene B, Hebbar M, Leteurtre E, van Seuningen I and Jonckheere N: The MUC4 mucin mediates gemcitabine resistance of human pancreatic cancer cells via the Concentrative Nucleoside Transporter family. Oncogene 32: 1714-1723, 2013.

16 Mimeault M, Johansson SL, Senapati S, Momi N, Chakraborty S and Batra SK: MUC4 down-regulation reverses chemoresistance of pancreatic cancer stem/progenitor cells and their progenies. Cancer Lett 295: 69-84, 2010

17 Wissniowski TT, Meister S, Hahn EG, Kalden JR, Voll R and Ocker M: Mucin production determines sensitivity to bortezomib and gemcitabine in pancreatic cancer cells. Int J Oncol 40: 1581$1589,2012$.

18 Urey C, Andersson B, Ansari D, Sasor A, Said-Hilmersson K, Nilsson J and Andersson R: Low MUC4 expression is associated with survival benefit in patients with resectable pancreatic cancer receiving adjuvant gemcitabine. ScandJ Gastroenterol 52: 595-600, 2017.

19 de Sousa Cavalcante L and Monteiro G: Gemcitabine: metabolism and molecular mechanisms of action, sensitivity and chemo-resistance in pancreatic cancer. Eur J Pharmacol 741: 8-16, 2014

20 Dubey RD, Saneja A, Gupta PK and Gupta PN: Recent advances in drug delivery strategies for improved therapeutic efficacy of gemcitabine. Eur J Pharm Sci 93: 147-162, 2016. 
21 Reid JM, Qu W, Safgren SL, Ames MM, Krailo MD, Seibel NL, Kuttesch $\mathrm{J}$ and Holcenberg J: Phase I trial and pharmacokinetics of gemcitabine in children with advanced solid tumors. J Clin Oncol 22: 2445-2451, 2004.

22 Dasanu CA: Gemcitabine: vascular toxicity and prothrombotic potential. Expert Opinion on Drug Safety 7: 703-716, 2008.

23 Kiani A, Köhne C-H, Franz T, Passauer J, Haufe T, Gross P, Ehninger $\mathrm{G}$ and Schleyer E: Pharmacokinetics of gemcitabine in a patient with end-stage renal disease: effective clearance of its main metabolite by standard hemodialysis treatment. Cancer Chemother Pharmacol 51: 266-270, 2003.

24 Mackey JR, Mani RS, Selner M, Mowles D, Young JD, Belt JA, Crawford CR and Cass CE: Functional nucleoside transporters are required for gemcitabine influx and manifestation of toxicity in cancer cell lines. Cancer Res 58: 4349-4357, 1998.

25 Spratlin J, Sangha R, Glubrecht D, Dabbagh L, Young JD, Dumontet C, Cass C, Lai R and Mackey JR: The absence of human equilibrative nucleoside transporter 1 is associated with reduced survival in patients with gemcitabine-treated pancreas adenocarcinoma. Clin Cancer Res 10: 6956-6961, 2004.

26 Nordh S, Ansari D and Andersson R: hENT1 expression is predictive of gemcitabine outcome in pancreatic cancer: a systematic review. World J Gastroenterol 20: 8482-8490, 2014.

27 Greenhalf W, Ghaneh P, Neoptolemos JP, Palmer DH, Cox TF, Lamb RF, Garner E, Campbell F, Mackey JR, Costello E, Moore MJ, Valle JW, McDonald AC, Carter R, Tebbutt NC, Goldstein D, Shannon J, Dervenis C, Glimelius B, Deakin M, Charnley RM, Lacaine F, Scarfe AG, Middleton MR, Anthoney A, Halloran CM, Mayerle J, Olah A, Jackson R, Rawcliffe CL, Scarpa A, Bassi C, Buchler MW and European Study Group for Pancreatic C: Pancreatic cancer hENT1 expression and survival from gemcitabine in patients from the ESPAC-3 trial. J Natl Cancer Inst 106: djt347, 2014.

28 Barenholz Y: Doxil(R)--the first FDA-approved nano-drug: lessons learned. J Control Release 160: 117-134, 2012.

29 Boulikas T: Clinical overview on Lipoplatin ${ }^{\mathrm{TM}}$ : a successful liposomal formulation of cisplatin. Expert Opinion on Investigational Drugs 18: 1197-1218, 2009.

30 Zhang H: Onivyde for the therapy of multiple solid tumors. OncoTargets and therapy 9: 3001-3007, 2016.

31 Siekmeier R and Scheuch G: Systemic treatment by inhalation of macromolecules - principles, problems, and examples. J Physiol Pharmacol 59(Suppl 6): 53-79, 2008.

32 Torchilin VP: Recent advances with liposomes as pharmaceutical carriers. Nat Rev Drug Discov 4: 145-160, 2005.

33 Immordino ML, Dosio F and Cattel L: Stealth liposomes: review of the basic science, rationale, and clinical applications, existing and potential. Int J Nanomedicine 1: 297-315, 2006.

34 Liu D, Mori A and Huang L: Role of liposome size and RES blockade in controlling biodistribution and tumor uptake of GM1-containing liposomes. Biochim Biophys Acta 1104: 95$101,1992$.
35 Torchilin V: Antibody-modified liposomes for cancer chemotherapy. Expert Opin Drug Deliv 5: 1003-1025, 2008.

36 Deshpande PP, Biswas S and Torchilin VP: Current trends in the use of liposomes for tumor targeting. Nanomedicine (Lond) 8 : 1509-1528, 2013.

37 Kontermann RE: Immunoliposomes for cancer therapy. Curr Opin Mol Ther 8: 39-45, 2006.

38 Singh AP, Chaturvedi $\mathrm{P}$ and Batra SK: Emerging Roles of MUC4 in Cancer: A Novel Target for Diagnosis and Therapy. Cancer Research 67: 433-436, 2007.

39 Rothdiener M, Beuttler J, Messerschmidt SK and Kontermann RE: Antibody targeting of nanoparticles to tumor-specific receptors: immunoliposomes. Methods Mol Biol 624: 295-308, 2010.

$40 \mathrm{Xu} \mathrm{H}$, Paxton J, Lim J, Li Y, Zhang W, Duxfield L and Wu Z: Development of high-content gemcitabine PEGylated liposomes and their cytotoxicity on drug-resistant pancreatic tumour cells. Pharm Res 31: 2583-2592, 2014.

$41 \mathrm{Xu} \mathrm{H}$, Paxton JW and Wu Z: Development of Long-Circulating $\mathrm{pH}-$ Sensitive Liposomes to Circumvent Gemcitabine Resistance in Pancreatic Cancer Cells. Pharm Res 33: 1628-1637, 2016.

42 Andrianifahanana M, Moniaux N, Schmied BM, Ringel J, Friess $\mathrm{H}$, Hollingsworth MA, Buchler MW, Aubert JP and Batra SK: Mucin (MUC) gene expression in human pancreatic adenocarcinoma and chronic pancreatitis: a potential role of MUC4 as a tumor marker of diagnostic significance. Clin Cancer Res 7: 4033-4040, 2001.

43 Alkholief $M$ and Campbell RB: Investigating the role of mucin in the delivery of nanoparticles to cellular models of human cancer disease: an in vitro study. Nanomedicine 12: 1291-1302, 2016.

44 Torres MP, Chakraborty S, Souchek J and Batra SK: Mucinbased targeted pancreatic cancer therapy. Curr Pharm Des 18: 2472-2481, 2012.

45 Chaturvedi P, Singh AP, Moniaux N, Senapati S, Chakraborty S, Meza JL and Batra SK: MUC4 mucin potentiates pancreatic tumor cell proliferation, survival, and invasive properties and interferes with its interaction to extracellular matrix proteins. Mol Cancer Res 5: 309-320, 2007.

46 Singh AP, Moniaux N, Chauhan SC, Meza JL and Batra SK: Inhibition of MUC4 expression suppresses pancreatic tumor cell growth and metastasis. Cancer Res 64: 622-630, 2004.

47 Ansari D, Urey C, Hilmersson KS, Bauden MP, Ek F, Olsson R and Andersson R: Apicidin sensitizes pancreatic cancer cells to gemcitabine by epigenetically regulating MUC4 expression. Anticancer Res 34: 5269-5276, 2014.

Received March 16, 2017

Revised April 10, 2017

Accepted April 12, 2017 